Review

\title{
Genome Editing in C. elegans and Other Nematode Species
}

\author{
Takuma Sugi ${ }^{1,2}$
}

1 PRESTO, Japan Science and Technology Agency, Kawaguchi 332-0012, Japan

2 Institute for Integrated Cell-Material Sciences (WPI-iCeMS), Kyoto University, Yoshida-Honmachi, Sakyo-ku, Kyoto 606-8501, Japan; tsugi@icems.kyoto-u.ac.jp; Tel.: +81-75-383-2164; Fax: +81-75-383-2541

Academic Editor: Izuho Hatada

Received: 17 August 2015; Accepted: 15 February 2016; Published: 26 February 2016

\begin{abstract}
Caenorhabditis elegans, a $1 \mathrm{~mm}$ long free-living nematode, is a popular model animal that has been widely utilized for genetic investigations of various biological processes. Characteristic features that make $C$. elegans a powerful model of choice for eukaryotic genetic studies include its rapid life cycle (development from egg to adult in 3.5 days at $20^{\circ} \mathrm{C}$ ), well-annotated genome, simple morphology (comprising only 959 somatic cells in the hermaphrodite), and transparency (which facilitates non-invasive fluorescence observations). However, early approaches to introducing mutations in the C. elegans genome, such as chemical mutagenesis and imprecise excision of transposons, have required large-scale mutagenesis screens. To avoid this laborious and time-consuming procedure, genome editing technologies have been increasingly used in nematodes including C. briggsae and Pristionchus pacificus, thereby facilitating their genetic analyses. Here, I review the recent progress in genome editing technologies using zinc-finger nucleases (ZFNs), transcriptional activator-like nucleases (TALENs), and clustered regularly interspaced short palindromic repeats (CRISPR)/Cas9 in nematodes and offer perspectives on their use in the future.
\end{abstract}

Keywords: nematode; C. elegans; transposon; TALEN; CRISPR/Cas9; cell-specific analysis

\section{Introduction}

The nematode C. elegans became a powerful model organism just over 50 years ago [1]. This tiny animal has greatly contributed to investigations of the functions of genes in development and cellular biology. One advantage of using C. elegans as a model animal is its simple morphology; the adult hermaphrodite is composed of only 959 somatic cells, including 302 neurons, and cell number does not vary among individuals. Researchers have tracked the fate of every cell, from fertilization to adulthood, in live animals and have generated a complete cell lineage [2]. This insight into cell lineage has contributed enormously to research in the field of developmental biology. The wiring and connectivity of neurons in C. elegans have been completely reconstructed from electron micrographs [3]. Therefore, this well-defined nervous system simplifies investigations of the neural circuitry underlying behavioral plasticity. Features that make the nematode highly amenable to laboratory research also include its small size, short life cycle and ability to survive long-term freezing for storage. These advantageous features have allowed C. elegans researchers to uncover novel biological mechanisms, such as apoptosis, and develop novel techniques, such as green fluorescent protein (GFP) tagging, in the life sciences research field.

C. elegans is a genetically tractable model system, for which many genetic resources and tools have been developed. There are approximately 20,359 protein-coding genes in C. elegans (WormBase referential freeze WS250, November 2015). In OrthoList, a compendium of C. elegans genes with 
human orthologs, 7663 protein-coding genes are predicted to have human orthologs, corresponding to approximately $38 \%$ of the C. elegans genome [4]. Furthermore, $60 \%-80 \%$ of human genes are represented by an ortholog in the C. elegans genome [5]. More importantly, $40 \%$ of genes known to be associated with human diseases are represented by orthologs in the C. elegans genome [6].

To clarify functional roles of $C$. elegans genes, various genetic methods have been developed and adapted before emergence of recent genome engineering techniques. In forward genetic screening, worms are treated with mutagens such as ethyl methane sulfonate (EMS) to induce DNA lesions and mutants with a target phenotype. Subsequent gene mapping and detailed analyses of the mutant phenotype allow elucidation of the gene's function. As a complement to forward genetics, reverse genetics offers opportunities to examine the function of a gene by analyzing the phenotypic effects of targeted gene sequence alteration. RNA interference (RNAi) has served as a powerful reverse genetic tool for large-scale genetic screening and to study gene function by examining the consequences of targeted gene knock-down. However, RNAi fails to completely suppress gene expression and provides only temporally inhibition of gene function only temporally. Screening of worms with deletions of specific target DNA by the C. elegans Gene Knockout Consortium and the Japan National Bioresource Project has offered opportunities to examine the phenotypic effects of a targeted gene knock-out. However, these resources do not completely include mutants with deletions in a desired genomic region. Therefore, it has been needed to establish a strategy for inducing targeted gene knock-out at will.

In compatible with forward and reverse genetic screening, microinjection of a gene of interest has been an indispensable genetic technique to study gene functions in C. elegans. The microinjection of genes including fluorescent protein-coding genes into the gonad of the adult hermaphrodite results in the formation of semi-stable extrachromosomal arrays that are comprised of many copies of the injected DNA [7]. The microinjection under the control of a cell-specific promoter has been used for an extrachromosomal rescue experiment that enables identification of a cell-specific role of the transgene. The transgenes, which are typically overexpressed in somatic tissues and silenced in the germline and early embryo, can be integrated into the genome by UV (ultraviolet) irradiation or microparticle bombardment [8,9]. These integration methods have allowed the generation of low-copy transgenes, which are expressed at closer to endogenous levels [10]. However, this experiment is less efficient, time-consuming, and not easily able to control genomic location for gene insertion. Thus, a strategy for inducing a stable gene knock-in at a desired genomic location has been required in the nematode research field.

Due to these requirements for targeted genome editing, C. elegans geneticists have developed methods based on the Mos1 transposon system [11,12]. Then, zinc-finger nucleases (ZFNs) [13], transcription activator-like effector (TALE) nucleases (TALENs) [14,15], and RNA-guided clustered regulatory interspaced short palindromic repeats (CRISPR)/Cas9 endonuclease systems have emerged as the revolutionized techniques adapted for creating locus-specific mutations in nematodes [15-22]. In this review, I provide a historical overview of this research field and discuss recent implementations of genome editing techniques in nematodes.

\section{Development of Gene Editing Protocols for Nematodes}

\subsection{Site-Restricted Editing by Mos1}

Before the revolution of the current genome editing technologies, methods based on the Mos1 transposon system allowed for targeted genome editing in C. elegans [11,12]. Robert and Bessereau reported that the Drosophila element Mos1 can also be used to produce double strand breaks (DSBs) and initiate gene editing [11]. In the experiments described in that paper, a repair template was constructed by preparing a transgene containing sequences homologous to the target genome region. The repair template was co-injected into the germline of a Mos1-insertion mutant with a vector enabling the expression of the Mos transposase using the heat-shock promoter $h s p-16.48$. Heat-shock treatment 
triggered DSBs at the target site, which were repaired by transgene-mediated gene conversion. This process allowed for mutations in the transgene to be copied at a specific locus at frequencies ranging from $10^{-5}$ to $10^{-4}$ events per F1 progeny. The mutations were successfully inherited by offspring. Frøkjær-Jensen et al. developed methods for Mos1-mediated single-copy insertion (MosSCI) that enables the insertion of single copies of transgenes into well-defined genomic loci [12] and optimized the efficiency of insertion [23]. The prerequisite for these techniques was the insertion of the Mos1 element into the target genomic region. Although efforts to generate a comprehensive Mos1-inserted mutant library are in progress, the ability to modify the genome with this technique is limited to genetic loci near transposons.

\subsection{Universal Editing with ZFNs, TALENs and CRISPRs}

Early methods for artificially generating DSBs at targeted genomic regions were based on ZFNs. ZFNs are chimeric proteins composed of DNA-binding $\mathrm{Cys}_{2} \mathrm{His}_{2}$ zinc finger motifs and a nonspecific FokI nuclease domain. Each finger is about 30 amino acids and interacts with a separate DNA triplet. Both natural and artificial zinc fingers have been characterized and known to bind to all 5'-GNN-3', many ANN and CNN, and some TNN triplets. In addition, the modular nature of the zinc fingers enables them to be assembled in arbitrary combinations. In general, three zinc fingers are combined to recognize a specific $9 \mathrm{bp}$ DNA sequence with nanomolar affinity, and additional fingers can be used to increase specificity. DSBs require the dimerization of two FokI nuclease domains, and ZFNs are used in pairs with specificity to opposing DNA strands, which enables assembly on both sides of the targeted site. This property enhances the specificity of ZFN targeting. Because zinc fingers exist for many of the DNA triplets, varying the number and types of fingers in each chimera would theoretically allow for targeting nucleases to the desired genomic loci. Carroll and colleagues initially designed a synthetic target DNA sequence for a previously characterized ZFN [13]. The synthetic target DNA along with the plasmid expressing the nuclease with a heat-shock promoter were co-injected into germline cells to generate an extrachromosomal array. Expression of the nuclease by heat-shock treatment induced mutations in more than $20 \%$ of the target sites. In subsequent experiments, an endogenous genomic sequence was targeted using a pair of designed ZFNs. This experiment achieved mutations in approximately $20 \%$ of the target sites by heat-shock induction. This study was the first to demonstrate that custom-engineered ZFNs could effectively generate targeted DSBs in nematode DNA. Although the repair of these breaks by the end-joining mechanism often generated mutations at the targeted locus, the procedure had little practical value as a genetic tool because the mutations were generated in somatic cells and hence were not inherited by offspring. Thus, these studies did not achieve the goal of heritable genome editing using ZFNs. The authors proposed that direct injection of mRNA into the germline might be effective for producing inherited mutations.

In a landmark paper on genome editing released in 2011, Wood et al. demonstrated heritable genome editing using ZFNs in both C. elegans and C. briggsae [14]. In the experiments described in this paper, the endogenous gene ben-1 was initially targeted because the phenotype of ben-1 heterozygous mutants can be scored easily by analyzing mobility on the paralysis-inducing drug benomyl. To attain heritable mutations in C. elegans, the investigators set the precedent of using mRNA, rather than DNA, delivery for getting germline production. As a proof of principle, the authors injected gonads with ZFN-encoding mRNAs carrying $5^{\prime}$ and $3^{\prime}$ untranslated regions amenable to germline translation and obtained ben-1/+ mutants at a frequency of approximately 3\% in F1 progeny. To establish a strategy for identifying mutants by molecular genotyping rather than relying on a visible phenotype, they targeted the REX-1 locus; REX-1 recruits the dosage compensation complex (DCC), thereby reducing gene expression. Using the CEL-1 assay, the authors isolated 18 independent mutant lines from 338 CEL-1 reactions, indicating that molecular genotyping was an effective method for phenotypic selection.

In TALENs, a nonspecific FokI nuclease domain is fused to a customizable TALE domain which contains tandem repeats of 33 or 34 amino acid segments and recognizes a predictable DNA sequence [24]. Residues 12 and 13 of the segment, referred to as repeat variable diresidues (RVDs), 
determine the base-specific binding (HD to C, NI to A, NG to T, NN to G) $[25,26]$. Sequence-specific DNA recognition by large TALE domains directs the nuclease, and the nuclease introduces DSBs at a prospective target site. Erroneous repair by end-joining often yields a mutagenic deletion or insertion at this breakpoint [27]. Compared with that of ZFNs, the recognition sequence of TALEs is relatively simple, and TALEs can be designed to bind to almost any sequence. Moreover, TALENs also bind as pairs, which also enhances specificity like ZFNs do. These features of TALEs are advantageous for many researchers who use genome editing techniques.

Woods et al. first utilized TALENs in nematodes as reported in their paper describing ZFNs [14]. TALEN-encoding mRNAs carrying $5^{\prime}$ and $3^{\prime}$ untranslated regions designed to target the ben-1 gene were injected into the C. elegans germline [14]. This method resulted in many TALEN-induced ben-1 mutations at a frequency of $1.1 \%$ in $\mathrm{F} 1$ progeny, and the same approach has been applied to C. species 9 (now called C. nigoni; $2.4 \%$ frequency) and P. pacificus (6.8\% frequency) as described in a subsequent paper [15]. Wei et al. have also used TALENs in C. briggsae and C. tropicalis [28,29]. The isolation of targeted mutants in C. elegans has typically relied on visible phenotypes or fluorescent markers such as GFP. In addition, molecular genotyping using CEL-1 assays [14], polyacrylamide electrophoresis [28], and heteroduplex mobility assays [30] has also been used to identify mutations that are not associated with a visible phenotype.

Although genome editing using TALENs has almost completely overcome the limitations associated with some target sequences, the technique requires the laborious custom design of TALE repeat segments. The CRISPR/Cas9 system emerged as a conceptually simple method that can be used as an alternative to TALENs [31-33]. In bacteria and archaea, the CRISPR locus functions with Cas proteins to facilitate an adaptive immune system response against invading foreign DNA, such as viral DNA or plasmids. The foreign nucleotides are incorporated into the CRISPR locus in the host genome, which results in short CRISPR RNAs (crRNAs) that direct the sequence-specific cleavage of homologous target dsDNA by Cas endonucleases. Previous studies with the type II CRISPR/Cas system, which requires the nuclease Cas9, a targeting crRNA and an additional trans-activating crRNA, have revealed that fusing the targeting and trans-activating RNAs to form a single guide RNA (sgRNA) is sufficient to direct Cas9-mediated target cleavage [31]. This system has been used for genome editing in other model organisms because of the simplicity in designing a sgRNA.

Friedland et al. initially constructed a plasmid that drives the expression of the gene encoding Cas9 using the germline-specific eft-3 promoter [16]. In that paper, $k l p-12$ and Y61A9LA.1, genes with an unknown loss-of-function phenotype, were targeted. The researchers isolated $k l p-12$ and Y 61 A9LA.1 mutations in $80.3 \%$ and $18.1 \%$ of the F1 worms screened, respectively. With respect to the klp-12 locus, 27 of $80 \mathrm{~F} 1$ worms carried a homozygous mutation. This mutation frequency is high enough to preclude the requirement of an obvious phenotype or visible selection marker. Like the TALEN system, the CRISPR system has been also proven to be useful for researchers working with non-Caenorhabditis nematodes. Witte et al. have successfully used CRISPR system-mediated gene inactivation in P. pacificus [34]. Thus, the CRISPR system is undoubtedly a versatile method to facilitate genome editing in the nematode.

Other research groups have also developed useful protocols and have demonstrated that heritable changes in nematode genes can be induced using the CRISPR/Cas9 system [15,17-22,35]. The protocols used in these experiments primarily differ with respect to delivery of the CRISPR/Cas9 effector complex (DNA, RNA, or protein) and the methods used to identify mutations (PCR screen, visible markers, or obvious phenotypes). For these experiments, most of the research groups expressed Cas 9 under the control of the eft-3 promoter. On the other hand, Boxem's group found the possibility that expression of Cas 9 from the eft-3 promoter causes embryonic lethality. The authors expressed Cas 9 using a heat-shock promoter within a limited time frame to prevent the lethality effects observed with the use of the eft-3 promoter and indicated that the use of the heat-shock promoter for Cas 9 expression might be better than that of the eft-3 promoter [21]. Other laboratories have used Cas9 and sgRNAs transcribed in vitro $[17,19]$ or crRNAs and tracrRNAs transcribed separately in vitro [15]. In one unique 
protocol, similarly to the RNAi feeding strategy, sgRNA was delivered to worms via bacterial feeding to achieve gene disruptions in a time- and labor-saving manner [36]. In this protocol, worms carrying pie-1p::Cas9 DNA were fed HT115 bacteria transformed with sgRNA. The mutation rates observed for the bli and dpy loci in this feeding protocol were low compared with the injection method, at $1.40 \%$ and $0.92 \%$, respectively. However, the authors have proposed that the protocols could potentially be redesigned to enable large-scale studies using a worm sgRNA library.

In brief, current ZFN, TALEN and CRIPSR systems have made possible important advances, but each has advantages and disadvantages in genome editing. In Zinc fingers, the context-dependency of the binding preference is that Zinc fingers exhibit context-dependent binding effects because of interactions between adjacent modules assembled into a larger array. In addition, assembly of functional ZFNs with the desired DNA-binding specificity remains a laborious process that requires extensive cell-based screening. In contrast, because TALE repeat domains exhibits fewer contextdependent preferences, researchers can simply assemble them in a modular fashion using a simple one-to-one code between individual repeats and the four possible DNA nucleotides. Frequency of off-target effects of TALENs is relatively low because of the length of the target sequence (15-19 bp), the higher specificity of the TALE DNA-binding domain, and the need of FokI dimerization to reconstitute endonuclease activity. On the other hand, despite these advantages over ZFNs, construction of novel TALE arrays still remains labor-intensive partly due to their repetitive sequences. In terms of target design simplicity, sgRNAs can be designed readily and cheaply to target nearly any genomic sequence specifically. Furthermore, the efficiency of the CRISPR system is high compared with the TALEN system. However, multiple mismatches between the guide RNA and its complementary target DNA sequence can be tolerated, thereby causing undesired off-target mutagenesis. Recent studies have demonstrated in other organisms that the use of dual nickases for Cas9 could reduce the potential off-target mutagenesis. Therefore, application of this strategy further revolutionizes the genome editing technology in the nematodes.

\subsection{Imprecise and Precise Repair Using ssOligo and dsDNA}

Genome editing techniques are a promising method for introducing DNA sequences encoding GFP or other tags and large-scale deletions at DSB sites. The methods for knock-in largely rely on the precise homology-dependent repair (HDR) of DSBs, in which a donor template that carries homology arms can be integrated during the repair process. Single-stranded oligonucleotides (ssOligos) and double-stranded DNA (dsDNA) including PCR fragments and plasmid DNAs, all of which contain homology arms, have been used as HDR donor templates [37]. ssOligos and PCR fragments can be easily designed and synthesized within a few days, and their use facilitated HDR-mediated knock-in. On the other hand, plasmid DNAs require cloning but can accommodate gene-sized edits and longer homology arms during repair processes. A previous evaluation of donor templates indicated that ssOligos are the preferred template in C. elegans, owing to their efficiency as well as ease of use (average frequencies for ssOligos and dsDNAs are 52\% and 20\%, respectively) [38]. In addition to HDR, other repair mechanisms also serve as inducing knock-ins and deletions at the targeted genomic loci. Van Schendel et al. have described an unexpected finding that while somatic cells use non-homologous end-joining (NHEJ), germ cells use exclusively polymerase theta-mediated end-joining (TMEJ), a simple repair mechanism requiring only one nucleotide as a repair template [39]. The researchers have demonstrated that polymerase theta is solely responsible for the vast majority of insertions and deletions that occur during the natural evolution of $C$. elegans. In addition, the imprecise end-joining repairs as well as template-mediated imprecise and precise HDRs have induced deletion mutations of variable length at the site of the DSB.

Lo et al. have demonstrated for the first time that TALENs can be used to introduce precise changes at endogenous sites using HDR templates [15]. This study was the first evidence that engineered nucleases and exogenous ssOligos as repair templates could be used to achieve heritable changes consisting of single nucleotide changes or insertions (e.g., HA tag) through precise HDR in nematodes 
at frequencies ranging from $0.2 \%$ to $4.0 \%$. Additionally, FLP recombinase was introduced into the strains possessing FRT sites flanking a cis-acting regulatory motif to knock out $\sim 1 \mathrm{~kb}$ of DNA, resulting in the removal of all the cis-regulatory information. By using an ssOligo, Lo et al. also demonstrated precise insertion of the HA tag gene in P. pacificus (1.0\% frequency). These results were the first to demonstrate tagging of an endogenous gene in a manner leaving no detectable marks other than the tag.

The CRISPR/Cas9 system has been also used with HDR templates. Dickinson et al. has generated HDR-mediated in-frame $g f p$ insertions and targeted mutations with an average frequency of $22 \%$ [40]. The authors constructed an HDR template comprising the C-terminal $1.5 \mathrm{~kb}$ sequence of the $n m y$ - 2 gene (illustrated as Gene A in Figure 1) fused in-frame to $g f p$, the $n m y-23^{\prime}$ UTR, and the unc-119(+) selection marker along with $1.5 \mathrm{~kb}$ of downstream genomic sequence (Figure 1) [40]. The unc-119(+) gene was flanked by loxP sites, which allowed for the excision of this gene by expression of Cre recombinase. At nearly the same time, Katic et al. also demonstrated transgene-induced gene conversion of the daf-2(m579) allele through Cas9-induced DSB repair by a wild-type sequence [19]. The investigators constructed a sgRNA targeting a $20 \mathrm{bp}$ sequence in daf-2 that overlapped with the $m 579$ mutation. The sgRNA was co-injected with a plasmid bearing the $715 \mathrm{bp}$ wild-type daf-2 sequence, which causes a missense change from $m 579$ into the wild-type sequence [19]. Instead of the plasmid, ssOligos and PCR fragments with short ( 30-60 bases) homology arms was previously examined as a donor template [41]. This micro-homology arm also enabled gene conversion and generated precise nucleotide changes via the CRISPR/Cas9 system. Furthermore, a variety of sequences encoding protein tags, such as V5, 3xFlag, Myc, and OLLAS, with short homology arms can be inserted into the desired target site through precise HDRs [42].

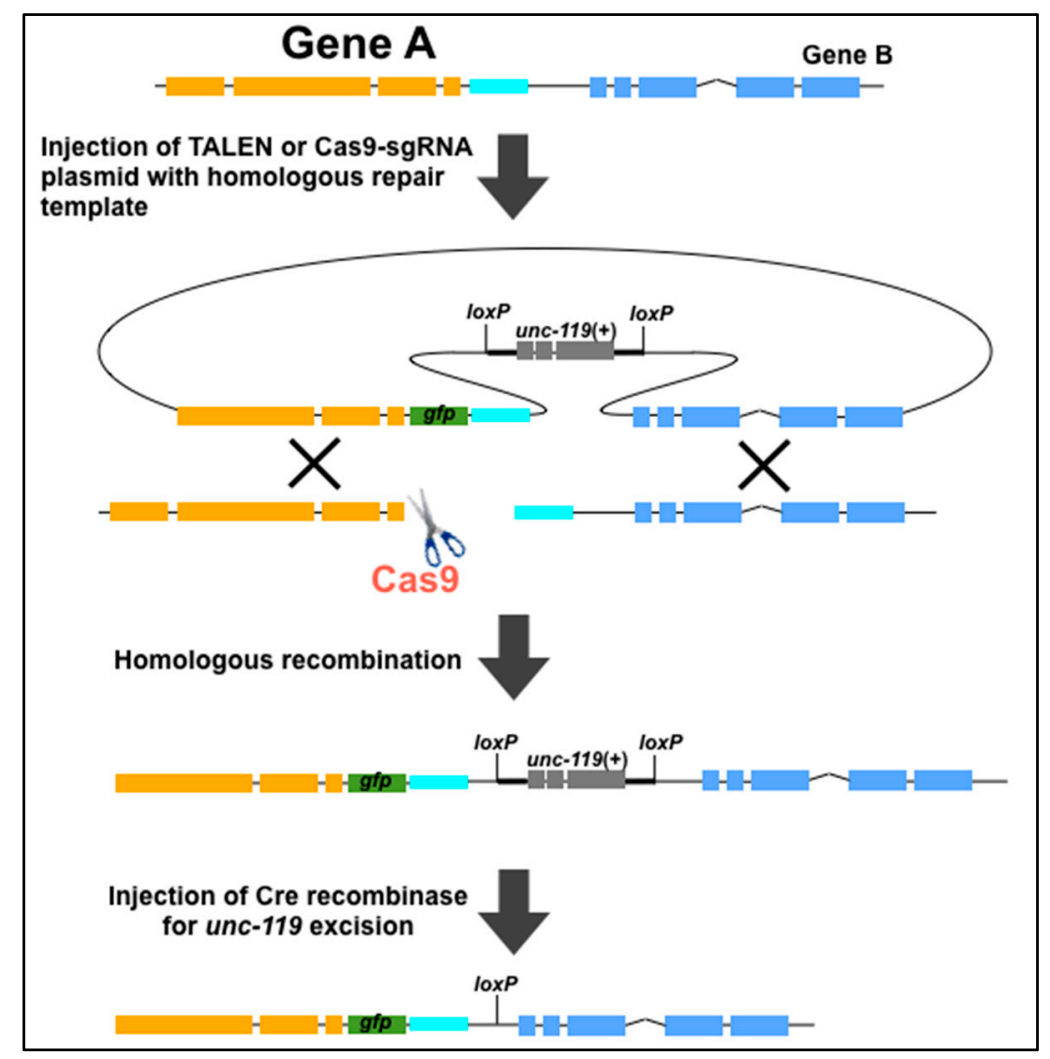

Figure 1. Schematic of homologous recombination-mediated $g f p$ insertion using TALENs or Cas9. The $u n c-119(+)$ selection marker in the resulting $g f p$-inserted worms can be excised by injecting Cre recombinase. The orange, blue, and cyan genomic regions are gene A, the 3' UTR of gene A, and gene B, respectively. The figure was adapted from Dickinson et al. [40]. 
In terms of deletion experiments, Lo et al. showed that deletions can be induced either immediately adjacent to the DSB by the end-joining mechanism (2.5\% frequency) or distant from the DSB by HDR (13.1\% frequency) using TALEN [15]. In another report, co-injection of worms with dual sgRNAs and Cas9 DNA has successfully induced the deletion of a chromosomal segment at least $24 \mathrm{~kb}$ in length [43]. Paix et al. have also reported that large deletions can be created directly by end-joining mechanism using Cas 9 and sgRNA pairs targeting the $5^{\prime}$ and $3^{\prime}$ ends of mbk-2 and the swan-1/swan-2 operon at $2.8 \%$ and $1.6 \%$ efficiencies, respectively [42]. Authors then examined whether ssOligos could be used to precisely create gene-length deletions by HDR and attempted to delete an entire ORF using an ssOligo targeting the K08F4.2 gene containing 51-80 bp micro-homology arms that flanked the sgRNA excision sites. Using this technique, they succeeded in generating large deletions with precise breakpoints in the K08F4.2 gene at a frequency of 3.3\%.

\section{Optimization of the CRISPR System}

Along with developments using the CRISPR system, several strategies for efficient identification of genome-edited worms have also been established in C. elegans. One such strategy is antibiotic resistance selection. Resistance to hygromycin B conferred by the HygR gene provides an efficient selection method in C. elegans. Thus, Chen et al. have combined this selection method with the CRISPR system and have created a HygR cassette flanked by $\sim 2 \mathrm{~kb}$ of DNA sequence homologous to either side of ben-1a sites [35]. CRISPR-based site-directed insertion of the HygR gene enabled hygromycin B-resistant selection of the desired ben-1 mutants. A toolkit composed of template-mediated repair cassettes that contain both an antibiotic resistance gene and a fluorescent visual maker, which facilitates identification of worms carrying the repair template, has also been developed [44].

Although these methods have facilitated the identification of specific mutants, a strategy that did not involve introducing an exogenous sequence at the targeted locus or marker mutations was still desired. Therefore, Dickinson et al. have designed a plasmid that includes both a fluorescent tag gene and the self-excising cassette (SEC) carrying a drug resistance gene, a visible phenotypic marker, and a heat-shock-inducible Cre recombinase gene [45]. Incorporation of the SEC from the plasmid into genomic loci permits easy excision of unwanted sequences upon heat-shock treatment after identification of the desired mutant using drug selection and screening for a visible phenotype.

Mello and colleagues have reported a "co-CRISPR" strategy that facilitates the identification of functional sgRNAs and increases the yield of transgenic worms carrying an end-joining or homologous recombination event (illustrated in Figure 2) [46]. The authors have found that approximately half of the sgRNAs tested are not effective in practice. This result is partly because conventional markers such as rol-6 result in false-positive mutant identification, and many injections fail to produce mutant progeny, even if high-quality reagents are used. Therefore, as an efficient marker, Mello et al. used an sgRNA targeting the muscle structural gene $u n c-22$, which has been previously proven to be active in generating CRISPR/Cas9-induced end-joining mutations, leading to an easily observable paralyzed twitching phenotype [47]. The selection based on the proven end-joining-induced phenotype would facilitate efficient identification of animals in which the CRISPR system was active. To examine this co-CRISPR strategy, the researchers co-injected the unc-22 sgRNA with targeted sgRNAs for avr-14 and $a v r-15$ or for pie-1b and pie-1c. Interestingly, selection based on the twitching phenotype dramatically increased the yield of animals in which a targeted sgRNA was active.

Arribere et al. have established a dominant phenotypic oligonucleotide template conversion strategy [48]. In this strategy (referred to as co-conversion strategy), instead of the sgRNA that induces end-joining-mediated mutations, an sgRNA and donor oligonucleotide to create the dominant phenotypic mutation such as rol-6(su1006) are co-injected with an sgRNA and donor oligonucleotide to create a mutation in the desired gene. By identifying the desired mutation among F1 progeny heterozygous for the dominant marker mutation, F2 worms that have lost the marker mutation can be isolated with the desired mutation in an unmarked genetic background. Arribere et al. have also identified one mutation in $d p y-10$ (cn64) and one mutation in sqt-1(e1350) as effective dominant markers. 
Recently, the repair of a temperature-sensitive lethal point mutation has been used as an alternate co-conversion marker [49]. This strategy promises robust selection, minimal screening, and has no requirement for out-crossing marker mutations.

In addition to these streamlined screening methods, another group has described the optimization of CRISPR target choices. A new sgRNA guide design strategy that vastly improved the efficiency of editing with Cas9 has been established [50]. The strategy uses sgRNA with a 3' GG addition to the target-specific sequence, which is key for improving the frequency of mutagenesis. When paired with the co-CRISPR approach, this technique offers the most reliable method of achieving precise genome editing in nematodes.

The CRISPR-mediated HDR protocols have also been improved in another manner. As an alternative to conventional protocols, the direct injection of recombinant Cas 9 protein complexed with guide RNA into C. elegans induces targeted gene disruption and yields HDR-mediated edits with frequencies ranging from $3.3 \%$ to $9.4 \%$ [18,51]. This strategy obviates the need to optimize DNA sequences for efficient expression of Cas9 and guide RNAs. Combined with the co-CRISPR strategy, Cas9/sgRNA ribonucleoprotein complexes have been proven more efficient and robust for use with low-efficiency guide RNAs and generating complex edits [51]. The author has established a direct-delivery protocol that increases the number of injected animals that produce mutant progeny (identified at frequencies ranging from $2 \%$ to $70 \%$ of $\mathrm{F} 1$ progeny), representing a 10 -fold improvement over conventional plasmid-based protocols.

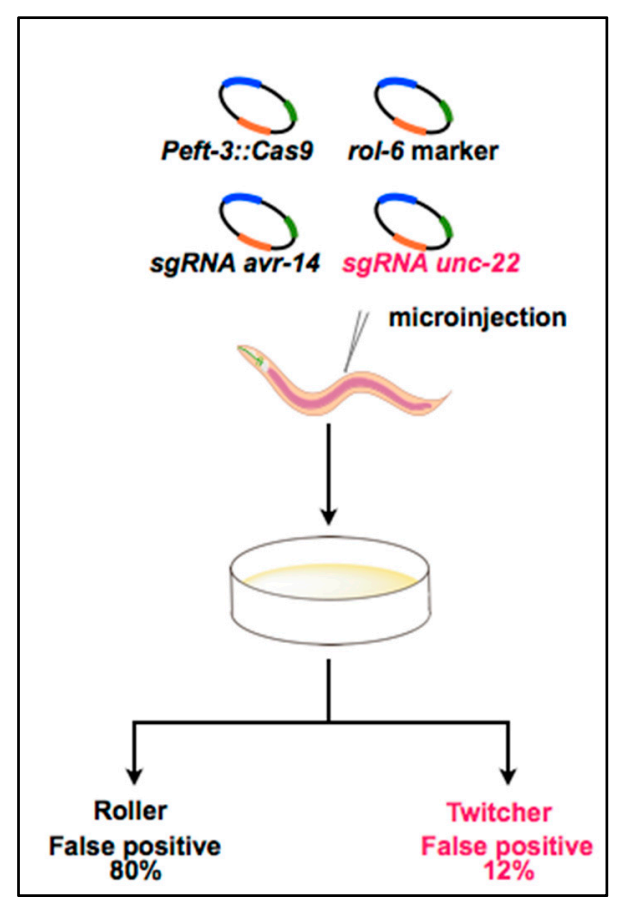

Figure 2. Overview of the co-CRISPR strategy for efficiently identifying functional sgRNAs targeting avr genes. sgRNA targeting unc-22, a gene associated with the twitcher phenotype, was co-injected with sgRNAs targeting avr-14 and avr-15, the Cas9 expression vector, and the rol-6 transformation marker. The co-injection of a functional unc-22 sgRNA reduced the false-positive rate in the identification of mutants. Figure 2 was adapted from Kim et al. [46].

\section{Use of Genome Editing Tools to Create New Methods for C. elegans}

In C. elegans, genetic studies can be conducted in a cell-specific manner. This advantage enables the conditional editing of a targeted sequence in the C. elegans genome. Conditional editing allows for the examination of genes associated with an embryonic lethal phenotype. Recently, Cheng et al. have developed a novel method to generate conditional knock-outs by expressing TALENs in somatic cells 
(referred to as somatic TALENs) [52]. The authors targeted the cor-1 gene, a worm ortholog of mouse actin-binding protein coronin. A cor-1 null mutation causes embryonic lethality, and its function was not known. The authors revealed that expression of a somatic TALEN targeting the cor- 1 under the Q-cell-specific promoter causes the migration defects of the AQR and PQR neurons that descended from the Q-cell in C. elegans larva, indicating the role of the cor-1 gene in Q-cell development. Thus, conditional knock-out was established by germline transformation with plasmids encoding TALENs using a cell-specific or inducible promoter. A similar strategy has also been established with the CRISPR system under the control of other cell-specific promoters. This strategy has helped to gain a further mechanistic insight into cor-1 [53] and to uncover roles of several other embryonic essential genes, including the dynein intermediate chain and a cytokinetic scaffold protein Anillin $[36,54,55]$.

In terms of conditional protein depletion, more recently, the auxin-inducible degradation (AID) system discovered in plants was applied to C. elegans [56]. It has been known through other model organisms that expression of an Arabidopsis TIR1 F-box protein mediates robust auxin-dependent degradation of degron-tagged targets by proteasomes. As proof of principle, the expression of TIR enabled rapid, reversible, auxin-dependent depletion of nuclear and cytoplasmic targets in diverse somatic and germline tissues throughout development in C. elegans.

Recently, Yumerefendi et al. have used Cas9-mediated homologous recombination with an interesting twist: a gene encoding light-activated protein was used as a repair template in place of tagged proteins [57]. The authors inserted a gene for light-activated nuclear shuttle proteins (referred to as LANS), which can change their nuclear localization upon light stimulation, into the C. elegans lin-1 locus via Cas9-mediated homologous recombination. A light-dependent functional switch of endogenous cellular transcription factor enabled optogenetic control of vulval cell fate specification in C. elegans.

CRISPR interference (CRISPRi), which has been developed to study sgRNA-mediated sequencespecific repression of transcription in prokaryotic and eukaryotic cells, was applied to C. elegans and D. rerio [58]. Long et al. revealed that transcription initiation and elongation of a gene can be interfered by the presence of the gRNA:DNA heteroduplex/dCas9 (a catalytically inactive form of Cas9) complex in its promoter and exons [58].

\section{Important Results Achieved Using Genome Editing Techniques}

Genome editing techniques have helped to identify biological functions of genes in C. elegans [56,59-61]. TALEN-mediated genome editing has been used for the targeted inactivation of the paxt-1 gene, which encodes a subunit of the XRN2 complex which is a eukaryotic exoribonuclease for processing and degrading various substrates [61]. This inactivation reduced XRN2 protein levels, decreased miRNA turnover, and caused lethality, suggesting that stabilization of XRN2 is a major function of PAXT-1.

Using gene editing, Ellis's group has addressed the molecular mechanisms underlying the evolution of self-fertile nematodes [29,62]. The investigators generated knock-out mutants of key genes involved in sperm activation in C. briggsae or C. tropicalis and found that C. briggsae, C. elegans, and C. tropicalis become self-fertile by co-opting one of the two redundant male programming pathways: the SPE- 8 tyrosine kinase pathway or the TRY-5 serine protease pathway. These pathways functioned redundantly in ancestral males [29]. Characterizations of an identified spe-47(hc198) allele using the CRISPR system showed that the $h c 198$ mutation is able to bypass the SPE-8 signaling pathway to cause premature activation of a small fraction of sperm [63]. In addition, the authors have found that the nucleosome remodeling factor (NURF) complex has acquired a unique role in the sperm/oocyte decision of C. briggsae [62]. This role was acquired during recent $C$. briggsae evolution.

Meyer and colleagues have used the CRISPR system to investigate $X$ chromosome dosage compensation, which balances gene expression between XX hermaphrodites and XO males [59]. Crane et al. used genome-wide chromosome conformation capture techniques to obtain three-dimensional (3D) maps of the C. elegans genome [59]. They initially revealed that the DCC remodels X chromosomes into a sex-specific spatial conformation distinct from autosomes. The dosage-compensated $\mathrm{X}$ chromosomes 
had been known to consist of self-interacting domains similar to topologically associated domains (TADs) in mammals. To address whether DCC-dependent interactions between sequence-specific recruitment elements on $X$ (rex sites) create TAD boundaries, they deleted the endogenous rex- 47 site from a DCC-dependent TAD boundary using the CRISPR system. This experiment shows that the DCC contributes to inducing and reinforcing TAD boundaries on the $\mathrm{X}$ chromosome by mediating long-range interactions between its highest affinity rex sites. This critical finding led the authors to conclude that the DCC dynamically remodels $\mathrm{X}$ chromosomes into a spatial conformation of TADs.

In addition to these achievements, genome editing has been a practical approach for investigating the role of microRNAs in vulval development [60]. Ecsedi et al. have found through editing the endogenous let-7 target site that, although let-7 has numerous targets including LET-60/RAS, it does not function to reduce gene expression noise broadly but instead functions to direct vulval development by regulating a single primary target, LIN-41/TRIM71 [60]. In terms of behavioral mechanism, Uozumi et al. found through proteomic screening that the mitochondrial voltage-dependent anion channel VDAC-1 functions as a candidate target of MAPK, and uncovered its role in olfactory behavior using the CRISPR system [64]. Thus, the practical application of genome editing techniques has facilitated the identification of novel roles for genes that had not been previously investigated by genetic experiments.

\section{Conclusions}

In nematode species, as with other model organisms, genome editing technologies have been used as indispensable tools for investigating biological systems. The previously available methods to generate mutations, such as chemical mutagenesis, use laborious procedures that require at least one month for large-scale mutagenesis screening, with unpredictable results. In contrast, recently developed genome editing techniques allow researchers to produce specific knock-out mutants or missense mutants in two to three weeks. Furthermore, they can conditionally create the targeted mutations with a cell-specific promoter. This technique provides an alternative avenue to investigate the role of a gene of interest in a cell-specific manner, avoiding the embryonic lethality caused by some mutations.

In other model organisms, TALE and Cas9 can be altered into a transcriptional regulator when they are fused to the transcriptional activator VP16/VP64 $[65,66]$ or to an epigenetic factor such as LSD1 histone demethylase [67]. Moreover, as an alternative to fluorescence in situ hybridization (FISH) methods, fluorescently tagged Cas9 labeling of specific DNA loci has been developed as a powerful live-cell imaging strategy [68]. These techniques should be highly compatible with C. elegans genetic methods, in which genes are expressed in only a target cell with a cell-specific promoter. Considering this remarkable feature, the causal roles of a gene expression change and its associated chromatin state at the single cell level can be studied in diverse biological processes including development, learning and memory, and disease. In summary, further promising techniques and novel biological concepts should emerge from the combination of genome editing technology and nematode cell-specific genetics.

Acknowledgments: The writing of this review was made possible in part by a grant from the Japan Society for the Promotion of Science, the Japan Science and Technology Agency under Precursory Research for Embryonic Science and Technology (PRESTO), and the Sumitomo Foundation.

Author Contributions: Takuma Sugi wrote this review.

Conflicts of Interest: The author declares no conflict of interest.

\section{References}

1. Brenner, S. The genetics of Caenorhabditis elegans. Genetics 1974, 77, 71-94. [PubMed]

2. Sulston, J.E.; Schierenberg, E.; White, J.G.; Thomson, J.N. The embryonic cell lineage of the nematode Caenorhabditis elegans. Dev. Biol. 1983, 100, 64-119. [CrossRef]

3. White, J.G.; Southgate, E.; Thomson, J.N.; Brenner, S. The structure of the nervous system of the nematode Caenorhabditis elegans. Philos. Trans. R. Soc. Lond. B Biol. Sci. 1986, 314, 1-340. [CrossRef] [PubMed] 
4. Shaye, D.D.; Greenwald, I. OrthoList: A compendium of C. elegans genes with human orthologs. PLoS ONE 2011, 6, e20085. [CrossRef] [PubMed]

5. Kaletta, T.; Hengartner, M.O. Finding function in novel targets: C. elegans as a model organism. Nat. Rev. Drug Discov. 2006, 5, 387-398. [CrossRef] [PubMed]

6. Culetto, E.; Sattelle, D.B. A role for Caenorhabditis elegans in understanding the function and interactions of human disease genes. Hum. Mol. Genet. 2000, 9, 869-877. [CrossRef] [PubMed]

7. Mello, C.C.; Kramer, J.M.; Stinchcomb, D.; Ambros, V. Efficient gene transfer in C. elegans: Extrachromosomal maintenance and integration of transforming sequences. EMBO J. 1991, 10, 3959-3970. [PubMed]

8. Praitis, V.; Casey, E.; Collar, D.; Austin, J. Creation of low-copy integrated transgenic lines in Caenorhabditis elegans. Genetics 2001, 157, 1217-1226. [PubMed]

9. Wilm, T.; Demel, P.; Koop, H.U.; Schnabel, H.; Schnabel, R. Ballistic transformation of Caenorhabditis elegans. Gene 1999, 229, 31-35. [CrossRef]

10. Sarov, M.; Murray, J.I.; Schanze, K.; Pozniakovski, A.; Niu, W.; Angermann, K.; Hasse, S.; Rupprecht, M.; Vinis, E.; Tinney, M.; et al. A genome-scale resource for in vivo tag-based protein function exploration in C. elegans. Cell 2012, 150, 855-866. [CrossRef] [PubMed]

11. Robert, V.; Bessereau, J.-L. Targeted engineering of the Caenorhabditis elegans genome following Mos1triggered chromosomal breaks. EMBO J. 2007, 26, 170-183. [CrossRef] [PubMed]

12. Frøkjaer-Jensen, C.; Davis, M.W.; Hopkins, C.E.; Newman, B.J.; Thummel, J.M.; Olesen, S.-P.; Grunnet, M.; Jorgensen, E.M. Single-copy insertion of transgenes in Caenorhabditis elegans. Nat. Genet. 2008, 40, 1375-1383. [CrossRef] [PubMed]

13. Morton, J.; Davis, M.W.; Jorgensen, E.M.; Carroll, D. Induction and repair of zinc-finger nuclease-targeted double-strand breaks in Caenorhabditis elegans somatic cells. Proc. Natl. Acad. Sci. USA 2006, 103, 16370-16375. [CrossRef] [PubMed]

14. Wood, A.J.; Lo, T.-W.; Zeitler, B.; Pickle, C.S.; Ralston, E.J.; Lee, A.H.; Amora, R.; Miller, J.C.; Leung, E.; Meng, X.; et al. Targeted genome editing across species using ZFNs and TALENs. Science 2011, 333, 307. [CrossRef] [PubMed]

15. Lo, T.-W.; Pickle, C.S.; Lin, S.; Ralston, E.J.; Gurling, M.; Schartner, C.M.; Bian, Q.; Doudna, J.A.; Meyer, B.J. Precise and heritable genome editing in evolutionarily diverse nematodes using TALENs and CRISPR/Cas9 to engineer insertions and deletions. Genetics 2013, 195, 331-348. [CrossRef] [PubMed]

16. Friedland, A.E.; Tzur, Y.B.; Esvelt, K.M.; Colaiácovo, M.P.; Church, G.M.; Calarco, J.A. Heritable genome editing in C. elegans via a CRISPR-Cas9 system. Nat. Meth. 2013, 10, 741-743. [CrossRef] [PubMed]

17. Chiu, H.; Schwartz, H.T.; Antoshechkin, I.; Sternberg, P.W. Transgene-free genome editing in Caenorhabditis elegans using CRISPR-Cas. Genetics 2013, 195, 1167-1171. [CrossRef] [PubMed]

18. Cho, S.W.; Lee, J.; Carroll, D.; Kim, J.-S.; Lee, J. Heritable gene knockout in Caenorhabditis elegans by direct injection of Cas9-sgRNA ribonucleoproteins. Genetics 2013, 195, 1177-1180. [CrossRef] [PubMed]

19. Katic, I.; Großhans, H. Targeted heritable mutation and gene conversion by Cas9-CRISPR in Caenorhabditis elegans. Genetics 2013, 195, 1173-1176. [CrossRef] [PubMed]

20. Tzur, Y.B.; Friedland, A.E.; Nadarajan, S.; Church, G.M.; Calarco, J.A.; Colaiácovo, M.P. Heritable custom genomic modifications in Caenorhabditis elegans via a CRISPR-Cas9 system. Genetics 2013, 195, 1181-1185. [CrossRef] [PubMed]

21. Waaijers, S.; Portegijs, V.; Kerver, J.; Lemmens, B.B.L.G.; Tijsterman, M.; van Den Heuvel, S.; Boxem, M. CRISPR/Cas9-targeted mutagenesis in Caenorhabditis elegans. Genetics 2013, 195, 1187-1191. [CrossRef] [PubMed]

22. Frøkjaer-Jensen, C. Exciting prospects for precise engineering of Caenorhabditis elegans genomes with CRISPR/Cas9. Genetics 2013, 195, 635-642. [CrossRef] [PubMed]

23. Frøkjaer-Jensen, C.; Davis, M.W.; Ailion, M.; Jorgensen, E.M. Improved Mos1-mediated transgenesis in C. elegans. Nat. Meth. 2012, 9, 117-118. [CrossRef] [PubMed]

24. Mussolino, C.; Cathomen, T. TALE nucleases: Tailored genome engineering made easy. Curr. Opin. Biotechnol. 2012, 23, 644-650. [CrossRef] [PubMed]

25. Boch, J.; Scholze, H.; Schornack, S.; Landgraf, A.; Hahn, S.; Kay, S.; Lahaye, T.; Nickstadt, A.; Bonas, U. Breaking the code of DNA binding specificity of TAL-type III effectors. Science 2009, 326, 1509-1512. [CrossRef] [PubMed]

26. Moscou, M.J.; Bogdanove, A.J. A simple cipher governs DNA recognition by TAL effectors. Science 2009, 326, 1501-1501. [CrossRef] [PubMed] 
27. Joung, J.K.; Sander, J.D. TALENs: A widely applicable technology for targeted genome editing. Nat. Rev. Mol. Cell Biol. 2013, 14, 49-55. [CrossRef] [PubMed]

28. Wei, Q.; Shen, Y.; Chen, X.; Shifman, Y.; Ellis, R.E. Rapid creation of forward-genetics tools for C. briggsae using TALENs: Lessons for nonmodel organisms. Mol. Biol. Evol. 2014, 31, 468-473. [CrossRef] [PubMed]

29. Wei, Q.; Zhao, Y.; Guo, Y.; Stomel, J.; Stires, R.; Ellis, R.E. Co-option of alternate sperm activation programs in the evolution of self-fertile nematodes. Nat. Commun. 2014, 5, 5888. [CrossRef] [PubMed]

30. Sugi, T.; Sakuma, T.; Ohtani, Y.; Yamamoto, T. Versatile strategy for isolating transcription activator-like effector nuclease-mediated knockout mutants in Caenorhabditis elegans. Dev. Growth Differ. 2014, 56, 78-85. [CrossRef] [PubMed]

31. Jinek, M.; Chylinski, K.; Fonfara, I.; Hauer, M.; Doudna, J.A.; Charpentier, E. A programmable dual-RNA-guided DNA endonuclease in adaptive bacterial immunity. Science 2012, 337, 816-821. [CrossRef] [PubMed]

32. Cong, L.; Ran, F.A.; Cox, D.; Lin, S.; Barretto, R.; Habib, N.; Hsu, P.D.; Wu, X.; Jiang, W.; Marraffini, L.A.; et al . Multiplex genome engineering using CRISPR/Cas systems. Science 2013, 339, 819-823. [CrossRef] [PubMed]

33. Mali, P.; Yang, L.; Esvelt, K.M.; Aach, J.; Guell, M.; DiCarlo, J.E.; Norville, J.E.; Church, G.M. RNA-guided human genome engineering via Cas9. Science 2013, 339, 823-826. [CrossRef] [PubMed]

34. Witte, H.; Moreno, E.; Rödelsperger, C.; Kim, J.; Kim, J.-S.; Streit, A.; Sommer, R.J. Gene inactivation using the CRISPR/Cas9 system in the nematode Pristionchus pacificus. Dev. Genes Evol. 2015, 225, 55-62. [CrossRef] [PubMed]

35. Chen, C.; Fenk, L.A.; de Bono, M. Efficient genome editing in Caenorhabditis elegans by CRISPR-targeted homologous recombination. Nucleic Acids Res. 2013, 41, e193. [CrossRef] [PubMed]

36. Liu, P.; Long, L.; Xiong, K.; Yu, B.; Chang, N.; Xiong, J.-W.; Zhu, Z.; Liu, D. Heritable/conditional genome editing in C. elegans using a CRISPR-Cas9 feeding system. Cell Res. 2014, 24, 886-889. [CrossRef] [PubMed]

37. Sander, J.D.; Joung, J.K. CRISPR-Cas systems for editing, regulating and targeting genomes. Nat. Biotechnol. 2014, 32, 347-355. [CrossRef] [PubMed]

38. Katic, I.; Xu, L.; Ciosk, R. CRISPR/Cas9 Genome Editing in Caenorhabditis elegans: Evaluation of templates for homology-mediated repair and knock-ins by homology-independent DNA repair. G3 (Bethesda) 2015, 5, 1649-1656. [CrossRef] [PubMed]

39. Van Schendel, R.; Roerink, S.F.; Portegijs, V.; van Den Heuvel, S.; Tijsterman, M. Polymerase $\Theta$ is a key driver of genome evolution and of CRISPR/Cas9-mediated mutagenesis. Nat. Commun. 2015, 6, 7394. [CrossRef] [PubMed]

40. Dickinson, D.J.; Ward, J.D.; Reiner, D.J.; Goldstein, B. Engineering the Caenorhabditis elegans genome using Cas9-triggered homologous recombination. Nat. Meth. 2013, 10, 1028-1034. [CrossRef] [PubMed]

41. Zhao, P.; Zhang, Z.; Ke, H.; Yue, Y.; Xue, D. Oligonucleotide-based targeted gene editing in C. elegans via the CRISPR/Cas9 system. Cell Res. 2014, 24, 247-250. [CrossRef] [PubMed]

42. Paix, A.; Wang, Y.; Smith, H.E.; Lee, C.-Y.S.; Calidas, D.; Lu, T.; Smith, J.; Schmidt, H.; Krause, M.W.; Seydoux, G. Scalable and versatile genome editing using linear DNAs with microhomology to Cas9 Sites in Caenorhabditis elegans. Genetics 2014, 198, 1347-1356. [CrossRef] [PubMed]

43. Chen, X.; Xu, F.; Zhu, C.; Ji, J.; Zhou, X.; Feng, X.; Guang, S. Dual sgRNA-directed gene knockout using CRISPR/Cas9 technology in Caenorhabditis elegans. Sci. Rep. 2014, 4, 7581. [CrossRef] [PubMed]

44. Norris, A.D.; Kim, H.-M.; Colaiácovo, M.P.; Calarco, J.A. Efficient genome editing in Caenorhabditis elegans with a toolkit of dual-marker selection cassettes. Genetics 2015, 201, 449-458. [CrossRef] [PubMed]

45. Dickinson, D.J.; Pani, A.M.; Heppert, J.K.; Higgins, C.D.; Goldstein, B. Streamlined genome engineering with a self-excising drug selection cassette. Genetics 2015, 200, 1035-1049. [CrossRef] [PubMed]

46. Kim, H.; Ishidate, T.; Ghanta, K.S.; Seth, M.; Conte, D.; Shirayama, M.; Mello, C.C. A co-CRISPR strategy for efficient genome editing in Caenorhabditis elegans. Genetics 2014, 197, 1069-1080. [CrossRef] [PubMed]

47. Moerman, D.G.; Baillie, D.L. Genetic organization in Caenorhabditis elegans: Fine-structure analysis of the unc-22 gene. Genetics 1979, 91, 95-103. [PubMed]

48. Arribere, J.A.; Bell, R.T.; Fu, B.X.H.; Artiles, K.L.; Hartman, P.S.; Fire, A.Z. Efficient marker-free recovery of custom genetic modifications with CRISPR/Cas9 in Caenorhabditis elegans. Genetics 2014, 198, 837-846. [CrossRef] [PubMed]

49. Ward, J.D. Rapid and precise engineering of the Caenorhabditis elegans genome with lethal mutation co-conversion and inactivation of NHEJ repair. Genetics 2015, 199, 363-377. [CrossRef] [PubMed] 
50. Farboud, B.; Meyer, B.J. Dramatic enhancement of genome editing by CRISPR/Cas9 through improved guide RNA design. Genetics 2015, 199, 959-971. [CrossRef] [PubMed]

51. Paix, A.; Folkmann, A.; Rasoloson, D.; Seydoux, G. High Efficiency, homology-directed genome editing in Caenorhabditis elegans using CRISPR-Cas9 ribonucleoprotein complexes. Genetics 2015, 201, 47-54. [CrossRef] [PubMed]

52. Cheng, Z.; Yi, P.; Wang, X.; Chai, Y.; Feng, G.; Yang, Y.; Liang, X.; Zhu, Z.; Li, W.; Ou, G. Conditional targeted genome editing using somatically expressed TALENs in C. elegans. Nat. Biotechnol. 2013, 31, 934-937. [CrossRef] [PubMed]

53. Shen, Z.; Zhang, X.; Chai, Y.; Zhu, Z.; Yi, P.; Feng, G.; Li, W.; Ou, G. Conditional knockouts generated by engineered CRISPR-Cas9 endonuclease reveal the roles of coronin in C. elegans neural development. Dev. Cell 2014, 30, 625-636. [CrossRef] [PubMed]

54. Li, W.; Yi, P.; Ou, G. Somatic CRISPR-Cas9-induced mutations reveal roles of embryonically essential dynein chains in Caenorhabditis elegans cilia. J. Cell Biol. 2015, 208, 683-692. [CrossRef] [PubMed]

55. Tian, D.; Diao, M.; Jiang, Y.; Sun, L.; Zhang, Y.; Chen, Z.; Huang, S.; Ou, G. Anillin regulates neuronal migration and neurite growth by linking RhoG to the actin cytoskeleton. Curr. Biol. 2015, 25, 1135-1145. [CrossRef] [PubMed]

56. Zhang, L.; Ward, J.D.; Cheng, Z.; Dernburg, A.F. The auxin-inducible degradation (AID) system enables versatile conditional protein depletion in C. elegans. Development 2015, 142, 4374-4384. [CrossRef] [PubMed]

57. Yumerefendi, H.; Dickinson, D.J.; Wang, H.; Zimmerman, S.P.; Bear, J.E.; Goldstein, B.; Hahn, K.; Kuhlman, B. Control of protein activity and cell fate specification via light-mediated nuclear translocation. PLoS ONE 2015, 10, e0128443. [CrossRef] [PubMed]

58. Long, L.; Guo, H.; Yao, D.; Xiong, K.; Li, Y.; Liu, P.; Zhu, Z.; Liu, D. Regulation of transcriptionally active genes via the catalytically inactive Cas9 in C. elegans and D. rerio. Cell Res. 2015, 25, 638-641. [CrossRef] [PubMed]

59. Crane, E.; Bian, Q.; McCord, R.P.; Lajoie, B.R.; Wheeler, B.S.; Ralston, E.J.; Uzawa, S.; Dekker, J.; Meyer, B.J. Condensin-driven remodelling of $\mathrm{X}$ chromosome topology during dosage compensation. Nature 2015, 523, 240-244. [CrossRef] [PubMed]

60. Ecsedi, M.; Rausch, M.; Großhans, H. The let-7 microRNA directs vulval development through a single target. Dev. Cell 2015, 32, 335-344. [CrossRef] [PubMed]

61. Miki, T.S.; Richter, H.; Rüegger, S.; Großhans, H. PAXT-1 promotes XRN2 activity by stabilizing it through a conserved domain. Mol. Cell 2014, 53, 351-360. [CrossRef] [PubMed]

62. Chen, X.; Shen, Y.; Ellis, R.E. Dependence of the sperm/oocyte decision on the nucleosome remodeling factor complex was acquired during recent Caenorhabditis briggsae evolution. Mol. Biol. Evol. 2014, 31, 2573-2585. [CrossRef] [PubMed]

63. LaMunyon, C.W.; Nasri, U.; Sullivan, N.G.; Shaw, M.A.; Prajapati, G.; Christensen, M.; Elmatari, D.; Clark, J.N. A New Player in the spermiogenesis pathway of Caenorhabditis elegans. Genetics 2015, 201, 1103-1116. [CrossRef] [PubMed]

64. Uozumi, T.; Hamakawa, M.; Deno, Y.-K.; Nakajo, N.; Hirotsu, T. Voltage-dependent anion channel (VDAC-1) is required for olfactory sensing in Caenorhabditis elegans. Genes Cells 2015, 20, 802-816. [CrossRef] [PubMed]

65. Crocker, J.; Stern, D.L. TALE-mediated modulation of transcriptional enhancers in vivo. Nat. Meth. 2013, 10, 762-767. [CrossRef] [PubMed]

66. Konermann, S.; Brigham, M.D.; Trevino, A.E.; Hsu, P.D.; Heidenreich, M.; Cong, L.; Platt, R.J.; Scott, D.A.; Church, G.M.; Zhang, F. Optical control of mammalian endogenous transcription and epigenetic states. Nature 2013, 500, 472-476. [CrossRef] [PubMed]

67. Mendenhall, E.M.; Williamson, K.E.; Reyon, D.; Zou, J.Y.; Ram, O.; Joung, J.K.; Bernstein, B.E. Locus-specific editing of histone modifications at endogenous enhancers. Nat. Biotechnol. 2013, 31, 1133-1136. [CrossRef] [PubMed]

68. Chen, B.; Gilbert, L.A.; Cimini, B.A.; Schnitzbauer, J.; Zhang, W.; Li, G.-W.; Park, J.; Blackburn, E.H.; Weissman, J.S.; Qi, L.S.; et al. Dynamic imaging of genomic loci in living human cells by an optimized CRISPR/Cas system. Cell 2013, 155, 1479-1491. [CrossRef] [PubMed]

(C) 2016 by the author; licensee MDPI, Basel, Switzerland. This article is an open access article distributed under the terms and conditions of the Creative Commons by Attribution (CC-BY) license (http://creativecommons.org/licenses/by/4.0/). 